\title{
The Arcetri Spectral Code for Optically-Thin Plasmas
}

\section{BRUNELLA C. MONSIGNORI FOSSI ${ }^{1}$ AND MASSIMO LANDINI ${ }^{2}$}

\author{
${ }^{1}$ Arcetri Astrophysical Observatory, Florence, Italy \\ ${ }^{2}$ Department of Astronomy and Space Science, University of Florence, Italy
}

The Arcetri-95 spectral code for optically thin plasmas computes the continuum and line emission of the ions of the most abundant elements. It includes the most updated atomic models and the main atomic processes for ions from Fe IX to Fe XXIII and for ions of the Belike, C-like, and N-like isoelectronic sequences. The power emitted per unit emission measure is produced as a function of temperature and density.

Comparison with observations requires the knowledge of the differential emission measure (DEM) as a function of temperature. A numerical code evaluates the best DEM distribution that satisfies observations and theoretical predictions. The spectral code together with the DEM code allows to compute synthetic spectra for any specified temperature distribution model of the plasma.

\section{Introduction}

The X-ray-UV emission from optically thin astrophysical plasmas has been measured by a large number of space programs, with the aim to study both solar and celestial sources. Future space missions are achieving high spectral resolution and sensitivity. A number of theoretical evaluations of emission spectra have been made over the temperature range $10^{4}$ to $10^{8} \mathrm{~K}$ (Landini \& Monsignori Fossi 1970; Mewe 1972; Raymond \& Smith 1977; Kato 1976; Stern et al. 1978; Gaetz \& Salpeter 1983; Mewe et al. 1985; Landini \& Monsignori Fossi 1990). In the large majority of cases the line emissivity has been computed using the semi-empirical formula of the effective Gaunt factor which relates the collision strength to the oscillator strength, but now a large number of accurate theoretical computations for electron excitation rate of ions exists and the updated theoretical spectral codes are produced using the most accurate atomic data and complete evaluation of the level population for each ion (Doyle \& Keenan 1992; Monsignori Fossi \& Landini 1994a; Brickhouse et al. 1995b).

An upgrading of the Arcetri X-ray-EUV spectral code (Landini \& Monsignori Fossi $1990)$ is now available and allows to evaluate continuum and line emission. Because a large number of lines pertaining to iron falls in the X-ray-EUV spectral range we have started to review the atomic data for ions of iron and, at this time, we have added the Belike, C-like, and N-like isoelectronic sequences. A critical assessment of the evaluation of atomic models and main atomic processes (collisional excitation rates, radiative decays, ...) has been performed. Stationary balance has been assumed to compute the number density population for each ion and the intensity of the emission lines has been evaluated as a function of temperature and density. The detections of Be-like, C-like, N-like and iron lines in X-ray-EUV spectral range provide inputs for spectral diagnostics in very broad temperature $(5.0 \leq \log T \leq 8.0)$ and density $\left(10^{6} \leq \log \mathrm{Ne} \leq 10^{15} \mathrm{~cm}^{-3}\right)$ regimes and allow investigation of astrophysical plasmas in very different physical conditions. 


\section{The Atomic Processes}

\subsection{Ionization Balance}

In stationary conditions the degree of ionization of an element is obtained by equating the ionization and recombination rates that relate successive stage of ionization. The evaluation of the rates is performed following approaches similar to those used by Arnaud \& Rothenflug (1985) and is described in Landini \& Monsignori Fossi (1990, 1991). For the iron ions the computation of Arnaud \& Raymond (1992) is used.

\subsection{The Radiative Emission}

The computer code evaluates continuum and line emission. The continuum emission includes free-free, free-bound and two-photons decay from H-like ions (Landini \& Monsignori Fossi 1990). The line emission includes a large number of radiative transitions of all the ions of the most important elements.

The bound-bound emissivity (power per unit volume) is given by:

$$
P_{i, j}=N_{j}\left(X^{+m}\right) A_{j, i} \frac{h c}{\lambda_{i, j}} \quad \text { erg } \mathrm{cm}^{-3} \mathrm{~s}^{-1}
$$

where $A_{j, i}\left(\mathrm{~s}^{-1}\right)$ is the Einstein spontaneous emission coefficient; $N_{j}\left(X^{+m}\right)$ is the number density of the level $j$ of the ion $(+m)$ of the element $X$.

In low density plasmas the collisional excitation processes are generally faster than ionization and recombination timescales, therefore the collisional excitation is dominant over ionization and recombination in producing excited states. The number density population of level $\boldsymbol{j}$ must be calculated by solving the statistical equilibrium equations and including all the important collisional and radiative excitation and de-excitation mechanisms:

$$
N_{j}\left(N_{e} \Sigma_{i} C_{j, i}^{e}+N_{p} \Sigma_{i} C_{j, i}^{p}+\Sigma_{i<j} A_{j, i}\right)=\Sigma_{i} N_{i}\left(N_{e} C_{i, j}^{e}+N_{p} C_{i, j}^{p}\right)+\Sigma_{i>j} N_{i} A_{i, j}
$$

with $C_{j, i}^{e}$ and $C_{j, i}^{p}$ the electron and proton collisional excitation rates $\left(\mathrm{cm}^{-3} \mathrm{~s}^{-1}\right), C_{i, j}^{e}$ and $C_{i, j}^{p}$ the electron and proton collisional de-excitation rates $\left(\mathrm{cm}^{-3} \mathrm{~s}^{-1}\right)$.

The collisional excitation rate for a Maxwellian electron velocity distribution is given by:

$$
C_{i, j}^{e}=\frac{8.63 \times 10^{-6}}{T_{e}^{1 / 2}} \frac{\Upsilon_{i, j}\left(T_{e}\right)}{\omega_{i}} \exp \left(\frac{-\Delta E_{i, j}}{k T_{e}}\right)
$$

where $\omega_{i}$ is the statistical weight of level $i, k$ is the Boltzmann constant and $\Upsilon_{i, j}$ (the effective collision strength) is the thermally-averaged collision strength:

$$
\Upsilon_{i, j}\left(T_{e}\right)=\int_{0}^{\infty} \Omega_{i, j} \exp \left(-\frac{E}{k T_{e}}\right) d\left(\frac{E}{k T_{e}}\right)
$$

where $\Omega_{i, j}$ is the collision strength, which is related to the electron excitation cross section, and $E$ is the energy of the scattered electron.

In many cases the proton collisional excitation and de-excitation processes must be included. They become comparable with electron collisional processes only for transitions where $\Delta E_{i, j} \ll k T_{e}$. This happens, for instance, for transitions between fine structure levels at high temperatures. In our code, till now, the proton collisional excitation and de-excitation rates are neglected.

The solution of the electron-ion scattering problem is complex and takes a great deal of computing resources (for an overview see Mason \& Monsignori Fossi 1994). The main approximations used for electron-ion scattering are Distorted Wave (DW), Coulomb Bethe (CBe) and the more elaborate Close-Coupling (CC) approximation. In general DW is 
thought to be accurate to about $25 \%$ and $\mathrm{CC}$ to better than $10 \%$. Resonance structures can contribute significantly to the excitation rates, particularly for forbidden and intersystem lines. A lot of effort has recently been put into the assessment of published atomic data, in particular electron excitation rates (Lang 1994). Here the evaluation of the effective collision strengths over a Maxwellian distribution has been performed following a slight modification of the method suggested by Burgess \& Tully (1992) for critically examining the electron scattering data calculations (for details see Monsignori Fossi \& Landini 1994b).

\subsection{The Data Base}

An extensive review of the available atomic data has been performed following the suggestions of the Abingdon meeting (Lang 1994); the most updated atomic models have been selected for iron ions, Be-like, C-like, and $\mathrm{N}$-like ions. When available, the observed values for the energy of the levels have been used in order to make an easier comparison with the observations; the radiative decay probabilities and electronic collision strengths or effective collision strengths have been collected for any pair of levels. In the next sections we give a brief description of the Arcetri data base.

\subsubsection{Iron Ions}

Fe IX: The atomic model consists of 17 levels $\left(3 p^{6}{ }^{1} S, 3 p^{5} 3 d{ }^{3} P,{ }^{3} F,{ }^{1} D,{ }^{3} D,{ }^{1} F,{ }^{1} P\right.$, $3 p^{5} 4 s^{3} P,{ }^{1} P$ ). The collision strengths are computed by Fawcett \& Mason (1989), the observed energies and the radiative probability are from Flower $(1977 \mathrm{~b})$ and Fawcett $\&$ Mason (1991).

Fe X: The atomic model consists of 31 levels $\left(3 s^{2} 3 p^{5}{ }^{2} P, 3 s 3 p^{6}{ }^{2} S, 3 s^{2} 3 p^{4} 3 d{ }^{4} D,{ }^{4} F\right.$, $\left.{ }^{2} P,{ }^{4} P,{ }^{2} D,{ }^{2} F,{ }^{2} G,{ }^{2} D,{ }^{2} S,{ }^{2} P,{ }^{2} D\right)$. The collision strengths are computed by Mason (1975) and the observed energies are from Corliss \& Sugar (1985).

Fe XI: The atomic model consists of 47 levels $\left(3 s^{2} 3 p^{4}{ }^{3} P,{ }^{1} D,{ }^{1} S, 3 s 3 p^{5}{ }^{3} P,{ }^{1} P\right.$, $\left.3 s^{2} 3 p^{3} 3 d^{5} D,{ }^{3} D,{ }^{3} F,{ }^{1} S,{ }^{3} G,{ }^{1} G,{ }^{1} D,{ }^{3} P,{ }^{1} F,{ }^{3} S,{ }^{1} P, 3 p^{6}{ }^{1} S\right)$. The collision strengths and the radiative probabilities are computed by Mason (1975), the observed energies are from Behring et al. (1972), Bromage et al. (1977), and Doschek et al. (1976).

Fe XII: The atomic model consists of 29 levels $\left(3 s^{2} 3 p^{3}{ }^{4} S,{ }^{2} D,{ }^{2} P, 3 s 3 p^{4}{ }^{4} P,{ }^{2} S,{ }^{2} D,{ }^{2} P\right.$, $\left.3 s^{2} 3 p^{2} 3 d^{4} P,{ }^{2} F,{ }^{2} D,{ }^{2} P,{ }^{2} S\right)$. The effective collision strengths are computed by Tayal \& Henry (1986), Tayal, Henry \& Pradhan (1987), the observed energies and the radiative probabilities are from Flower (1977a). Other radiative probabilities are from Bromage et al. (1978) and Tayal \& Henry (1988).

Fe XIII: The atomic model consists of 24 levels $\left(3 s^{2} 3 p^{2}{ }^{3} P,{ }^{1} D,{ }^{1} S, 3 s 3 p^{3}{ }^{5} S,{ }^{3} D,{ }^{3} P\right.$, $\left.{ }^{1} D,{ }^{3} S,{ }^{1} P, 3 s^{2} 3 p 3 d^{3} P,{ }^{1} D,{ }^{3} D,{ }^{1} P,{ }^{1} F\right)$. The collision strengths and the observed energies are from Fawcett \& Mason (1989). The radiative probabilities are from Mc Kim Melville \& Berger (1965), Bromage et al. (1978) and Corliss \& Sugar (1985).

Fe XIV: The atomic model consists of 12 levels $\left(3 s^{2} 3 p{ }^{2} P, 3 s 3 p^{2},{ }^{4} P,{ }^{2} D,{ }^{2} S,{ }^{2} P\right.$, $\left.3 s^{2} 3 d^{2} D\right)$. The effective collision strengths are from Dufton \& Kingston (1991), the observed energies are from Mason (1975). The radiative probabilities are from Froese, Fisher, \& Liu (1986).

Fe XV: The atomic model consists of 16 levels $\left(2 s^{2}{ }^{1} S, 3 s 3 p{ }^{3} P, P, 3 p^{2}{ }^{1} D,{ }^{3} P,{ }^{1} S, 3 s 3 d\right.$ $\left.3 s 4 s^{3} S,{ }^{1} S\right)$. The effective collision strengths, radiative probabilities and the observed energies are from Christensen et al. (1985) and Pradhan (1988).

Fe XVI: The atomic model consists of 19 levels $\left(3 s^{2} S, 3 p{ }^{2} P, 3 d{ }^{2} D, 4 s{ }^{2} S, 4 p{ }^{2} P\right.$, $4 d^{2} D, 4 f^{2} F, 5 s^{2} S, 5 p^{2} P, 5 d^{2} D, 5 f^{2} F$ ). The observed energies are from Corliss \& Sugar (1985); the oscillator strengths and collision strengths are from Sampson, Zhang, \& Fontes (1990). 
Fe XVII: The atomic model consists of 37 levels $\left(2 p^{6}{ }^{1} S, 2 p^{5} 3 s^{3} P,{ }^{1} P, 2 p^{5} 3 p{ }^{3} S\right.$, ${ }^{3} D,{ }^{3} P,{ }^{1} P,{ }^{1} D,{ }^{1} S, 2 p^{5} 3 d^{3} P,{ }^{3} F,{ }^{3} D,{ }^{1} D,{ }^{1} F,{ }^{1} P, 2 s 2 p^{6} 3 s{ }^{3} S,{ }^{1} S, 2 s 2 p^{6} 3 p^{3} P,{ }^{1} P$, $\left.2 s 2 p^{6} 3 d^{3} D,{ }^{1} D\right)$. The collision strengths, the radiative probabilities and the observed energies are from Bhatia \& Doschek (1992).

Fe XVIII: The atomic model consists of 108 levels $\left(2 p^{5}{ }^{2} P, 2 s 2 p^{6}{ }^{2} S, 2 p^{4} 3 s{ }^{4} P,{ }^{2} P\right.$, ${ }^{2} D,{ }^{2} S, 2 p^{4} 3 p{ }^{4} P,{ }^{4} D,{ }^{2} D,{ }^{2} P,{ }^{4} S,{ }^{2} S,{ }^{2} F, 2 p^{4} 3 d^{4} D,{ }^{4} F,{ }^{2} F,{ }^{2} D,{ }^{2} G,{ }^{2} S,{ }^{2} P,{ }^{4} P$, $\left.2 s 2 p^{5} 3 s{ }^{4} P,{ }^{2} P, 2 s 2 p^{5} 3 p{ }^{4} S,{ }^{4} D,{ }^{4} P,{ }^{2} P, 2 s 2 p^{5} 3 d{ }^{4} P,{ }^{4} D,{ }^{2} P,{ }^{2} D,{ }^{2} F,{ }^{2} S\right)$. The collision strengths, the radiative probabilities and the observed energies are from Cornille et al. (1992).

Fe XIX: The atomic model consists of 10 levels $\left(2 s^{2} 2 p^{4}{ }^{3} P,{ }^{1} D,{ }^{1} S, 2 s 2 p^{5}{ }^{3} P,{ }^{1} P\right.$, $2 p^{6}{ }^{1} S$ ). The observed energies, the collision strengths and the radiative probabilities are from Loulergue et al. (1985).

Fe XX: The atomic model consists of 13 levels $\left(2 s^{2} 2 p^{3}{ }^{4} S,{ }^{2} D,{ }^{2} P, 2 s 2 p^{4}{ }^{4} P,{ }^{2} D,{ }^{2} S\right.$, ${ }^{2} P$ ). The observed energies, the collision strengths and the radiative probabilities are from Bhatia \& Mason (1980a).

Fe XXI: The atomic model consists of 36 levels $\left(2 s^{2} 2 p^{2}{ }^{3} P,{ }^{1} D,{ }^{1} S, 2 s 2 p^{3}{ }^{5} S,{ }^{3} P\right.$, ${ }^{3} D,{ }^{3} S,{ }^{1} P,{ }^{1} D, 2 s^{2} 2 p 3 s{ }^{3} P,{ }^{1} P, 2 s^{2} 2 p 3 d{ }^{3} F,{ }^{1} D,{ }^{3} D,{ }^{3} P,{ }^{1} F,{ }^{1} P, 1 s^{2} 2 p^{4}{ }^{3} P$, $\left.{ }^{1} D,{ }^{1} S\right)$. The observed energies, the collision strengths and radiative probabilities are from Mason et al. (1979) for the first 31 levels. The effective collision strengths and the observed energies for the configuration $1 s^{2} 2 p^{4}$ are from Aggarwal (1991).

Fe XXII: The atomic model consists of 20 levels $\left(2 s^{2} 2 p{ }^{2} P,{ }^{4} P,{ }^{2} D,{ }^{2} S, 2 s 2 p^{2}{ }^{4} P,{ }^{2} D\right.$, $\left.{ }^{2} S,{ }^{2} D, 2 p^{3}{ }^{4} S,{ }^{2} D,{ }^{2} P, 2 p^{3}{ }^{4} S,{ }^{2} D,{ }^{2} P, 2 s^{2} 3 s^{2} S,{ }^{2} P, 2 s^{2} 3 p{ }^{2} P, 2 s^{2} 3 d^{2} D\right)$. The observed energies are from Edlen (1983) and Bhatia et al. (1986), collision strengths and radiative probabilities are from Bhatia et al. (1986).

Fe XXIII: The atomic model consists of 30 levels $\left(2 s^{2}{ }^{1} S, 2 s 2 p{ }^{3} P,{ }^{1} P, 2 p^{2}{ }^{3} P,{ }^{1} D,{ }^{1} S\right.$, $\left.2 s 3 s^{3} S,{ }^{1} S, 2 s 3 p^{3} P,{ }^{1} P, 2 s 3 d{ }^{3} D,{ }^{1} D, 2 s 4 s^{3} S_{1},{ }^{1} S, 2 s 4 p{ }^{3} P,{ }^{1} P, 2 s 4 d^{3} D,{ }^{1} D\right)$. The observed energies are from Bhatia \& Mason (1981), the collision strengths and the radiative probabilities are from Bhatia \& Mason (1986) for the first 20 levels. For the configurations $2 \mathrm{~s} 4 \mathrm{~s}, 2 \mathrm{~s} 4 \mathrm{p} 2 \mathrm{~s} 4 \mathrm{~d}$ the atomic data are supplied by Bhatia (private communication).

\subsubsection{Be-like Ions}

C III: The atomic model consists of 20 levels $\left(2 s^{2}{ }^{1} S, 2 s 2 p{ }^{3} P,{ }^{1} P, 2 p^{2}{ }^{3} P,{ }^{1} D,{ }^{1} S\right.$, $\left.2 s 3 p{ }^{3} P,{ }^{1} P, 2 s 3 d^{3} D,{ }^{1} D\right)$. The collisional strengths and the radiative probabilities are from Bhatia \& Kastner (1993a); the observed energies are from NIST energy levels database.

O V, Ne VII, Mg IX, Ca VII, Si XI, Ni XXV: The atomic model consists of 10 levels $\left(2 s^{2}{ }^{1} S, 2 s 2 p^{3} P,{ }^{1} P, 2 p^{2}{ }^{3} P,{ }^{1} D,{ }^{1} S\right)$. The collisional strengths and the radiative probabilities for allowed transitions are from Zhang \& Sampson (1992). For forbidden and intercombination transitions the radiative probabilities are from Muhlethaler \& Nussbaumer (1976) and Nussbaumer \& Storey (1979). The observed energies are from NIST energy levels database.

\subsubsection{C-like Ions}

The atomic model consists of 46 levels $\left(2 s^{2} 2 p^{2}{ }^{3} P,{ }^{1} D,{ }^{1} S, 2 s 2 p^{35} S,{ }^{3} D,{ }^{3} P, 2 s^{2} 2 p 3 d^{3} F\right.$, $O$ III: The collisional strengths and the radiative probabilities are from Bhatia \& Kastner (1993b). The observed energies are from Edlen (1985) and Wiese et al. (1966).

Ne V: The collisional strengths and the radiative probabilities are from Bhatia \& Doschek (1993a). The observed energies are from Edlen (1985) and Wiese et al. (1966). 
Mg VII: The collisional strengths and the radiative probabilities are from Bhatia \& Doschek (1995). The observed energies are from Edlen (1985) and Wiese et al. (1966). Si IX: The collisional strengths and the radiative probabilities are from Bhatia \& Doschek (1993b). The observed energies for the configurations $2 s^{2} 2 p^{2}, 2 s 2 p^{3}, 2 p^{4}$ are from Edlen (1985).

S XI: The collisional strengths and the radiative probabilities are from Bhatia et al. (1987). The observed energies are from Edlen (1985) and Wiese et al. (1966).

Ca XV: The collisional strengths and the radiative probabilities are from Bhatia \& Doschek (1993c) The observed energies for the configurations: $2 s^{2} 2 p^{2}, 2 s 2 p^{3}, 2 p^{4}$ are from Edlen (1985).

Zn XXV: The collisional strengths and the radiative probabilities are from Bhatia et al. (1987) The observed energies for the configurations: $2 s^{2} 2 p^{2}, 2 s 2 p^{3}, 2 p^{4}$ are from Edlen (1985).

\subsection{4. $N$-like Ions}

The atomic model consists of 13 levels $\left(2 s^{2} 2 p^{3}{ }^{4} S,{ }^{2} D,{ }^{2} P, 2 s 2 p^{4}{ }^{4} P,{ }^{2} D,{ }^{2} S,{ }^{2} P\right)$. The collisional strengths and the radiative probabilities are from Bhatia \& Mason (1980b). Mg VI, Si VII, S X: The observed energies are from Wiese et al. (1966).

Ar XII, Ca XIV: The observed energies are from Edlen (1984).

\section{Plasma Diagnostics}

The reason for studying astrophysical spectra is to determine the physical parameters of the plasma, such electron density, temperature distribution, element abundances and velocity fields. The first item to be addressed is to identify correctly the lines in the always higher resolution spectra. For this aim it is useful to produce the synthetic spectrum of the source using any temperature distribution model of the plasma.

\subsection{Differential Emission Measure Analysis}

Physical information on the source model are hidden in the emission measure, which is a quantity that any theoretical model should predict. The aim of temperature diagnostic is to extract such empirical information using a set of measured line intensities.

The following procedure has been developed (Monsignori Fossi \& Landini 1991):

- A set of density insensitive lines must be selected.

- The differential emission measure:

$$
\mathrm{DEM}=N_{e}^{2} \frac{d V}{d T}=f(T)
$$

is defined and assumption is made that it is a function of temperature only.

- The flux measured at Earth for each line $j$ is given by:

$$
I_{j}=\frac{1}{4 \pi D^{2}} \int_{V} G_{j}(T) N_{e}^{2} d V=\int_{T} G(T) f(T) d T
$$

where $D$ is the distance from the source to the Earth and $G(T)$ is the so-called contribution function, that takes into account the atomic data of the transition. A set of these equations are a system of integral equations of Frehdolm of first order.

- An inversion technique must be applied to evaluate the best $f(T)$ function which satisfies the observed lines.

It is particularly useful to compute the solution, following an iterative procedure in which the $f(T)$ is described by a cubic spline function through a small number $(n)$ of mesh 


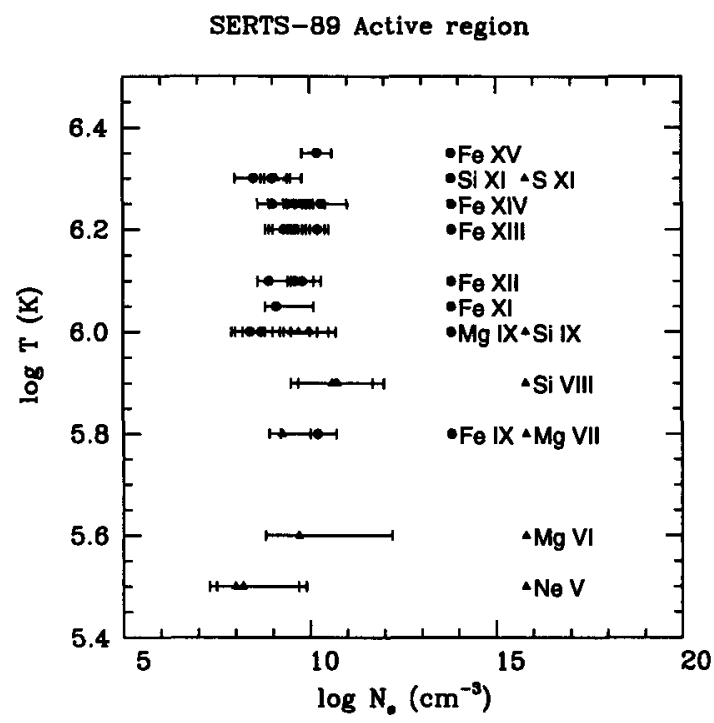

FIGURE 1. The EUVE spectrum of AT Microscopii. Top: the observed SW spectrum. Bottom: the synthetic spectrum (Monsignori Fossi et al. 1995b)

points $\left(f_{i}=f\left(T_{i}\right), i=1, \ldots, n\right)$ and $f_{i}$ are changed until the best fit is obtained. The $(r+1)$ approximation is achieved by the previous one $(r)$ using the recurrent expression:

$$
\ln f_{i}^{r+1}=\ln f_{i}^{r}-2 \lambda \Sigma_{j} \frac{\left(I_{e x, j}-I_{o b, j}\right)^{2}}{\sigma_{s}^{2}} \frac{\partial I_{e x, s}}{\partial f_{i}}
$$

where $I_{o b, j}$ is the observed signal, $I_{e x, j}$ is the expected signal, computed using the current $f^{r}(T)$ approximation, and $\lambda$ is a Lagrange multiplier.

This procedure gives only positive solutions, is usually quickly converging and allows to control oscillation or flattening through the number of mesh points. It can give large indetermination in the temperature intervals where poor constraints are put by the available observations. For this reason it is important to have an appropriate selection of the lines, specially with regard to their sensitivity to different temperatures. Using the measured DEM, the synthetic spectrum can been evaluated and used for a detailed comparison with the observation to get new identifications and a general check on the theory. This technique was applied to study the spectra recorded by the Extreme Ultraviolet Explorer (EUVE) spectrometers for the active stars AU Mic (Landini \& Monsignori Fossi 1993; Monsignori Fossi \& Landini 1994c), AT Mic (Monsignori Fossi et al. 1995b), EQ Peg (Monsignori Fossi et al. 1995a). In Figure 1 the result for the SW section spectrum of AT Mic is shown.

\subsection{Density Sensitive Lines: Comparison with Experimental Data}

The code can evaluate the line emissivities for temperatures between $10^{4}$ and $10^{8} \mathrm{~K}$ and densities between $10^{5}$ and $10^{16} \mathrm{~cm}^{-3}$. The $\mathrm{X}$-EUV spectral region is crowded by bright iron lines and Be-, C-, and N-like ions lines. Several lines of the most abundant element are useful to perform density diagnostic in astrophysical plasmas.

The electron density may be evaluated from the ratios of proper spectral line pairs pertaining to the same ion. If the source is homogeneous, this procedure does not make 

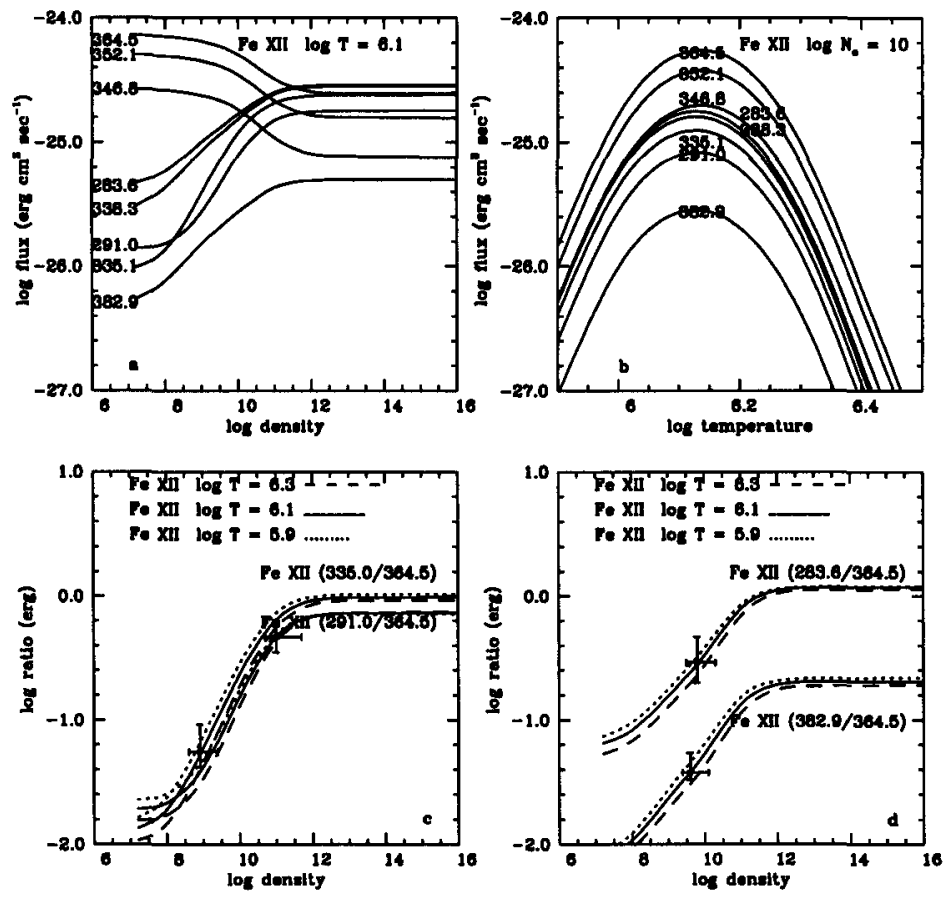

FIGURE 2. Fe XXI ratio useful to determine density in flares.

any assumption about the size of the emitting volume, the relative ion abundance and the elemental abundance.

A number of density sensitive lines were detected by the $E U V E$ spectrometers both in the spectra of active stars as Capella, AU Mic, EQ Peg, Algol (Dupree et al. 1993; Monsignori Fossi \& Landini 1994c; Monsignori Fossi et al. 1995a; Stern et al. 1995) and in the solar-like stars as $\alpha$ Cen, Procion (Mewe et al. 1995; Young et al. 1995). The densities evaluated during the large flare on the star AU Mic, observed by EUVE spectrometers, on July 1992 are shown in Figure 2 together with the density deduced in the OSO-5 solar flare. The stellar flare requires at any time density larger than observed in the solar flare.

\section{Comparison with Other Spectral Codes}

Comparison between different spectral codes for optically thin plasma has been recently performed (Brickhouse et al. 1995a). In the EUV spectral region, the agreement, between the codes, is rather good specially for high temperature regimes, where the main contribution to the emission comes from the high ionization stages of iron ions. The main differences of the examined codes concern the evaluation of the line emissivity and the spectral range. The Brickhouse et al. (1995a) code solves the statistical equations only for the iron ions; the Mewe et al. (1995) code computes the line emissivity with the Gaunt factor approximation and covers the spectral range below $1000 \AA$; the Masai code (Masai 1994) evaluates the emission only in the X-ray spectral region. The Arcetri-95 code covers the spectral region $1-3000 \AA$. For several ions the line's emissivity in the IR 
is also evaluated. The Arcetri code, at this time, seems the most complete, because it is updated with the most reliable atomic data for iron ions and $\mathrm{Be}, \mathrm{C}$ and $\mathrm{N}$ isoelectronic sequences ions and produces the power emitted per unit emission measure as function of temperature and density.

\section{Conclusions}

The best available atomic data for $\mathrm{Be}, \mathrm{C}$, and $\mathrm{N}$-like isoelectronic ions and for $\mathrm{Fe}$ IX to Fe XXIII have been used to evaluate level population under the balance among electron collisional excitations and de-excitation and radiative decay and inserted in the updated version of the Arcetri spectral code. It produces synthetic spectra of plasma emission for temperatures $(4.0 \leq \log T \leq 8.0)$ and densities $\left(10^{5} \leq \log \mathrm{Ne} \leq 10^{15}\right)$. A numerical procedure to evaluate empirical DEM distribution together with the theoretical spectrum allows one to compute the synthetic spectra which recover the observations.

An updated version of Arcetri spectral code (January 1995) in the wavelength range $60-760 \AA$ is available for free distribution via WWW (http://www.arcetri.astro.it). In the future we will provide on WWW the updated version for the soft X-ray spectral region including the $B$-like ions of the most abundant elements.

We would like to acknowledge Drs. Bhatia and Zhang for providing their computations. The research was supported by the Italian Space Agency (ASI).

\section{REFERENCES}

Aggarwal, K. M. 1991, ApJS, 77, 677

ARNAUD, M. \& Rothenflug, R. 1985, A\&AS, 60, 425

ARNAUD, M. \& RAYMOND, J. C. 1992, A\&AS, 398, 394.

Behring, W. E., Cohen, L. \& Feldman, U. 1972, ApJ, 175, 493

Behring, W. E., Cohen, L. Feldman, U., \& DoscheK, G. A. 1976, ApJ, 203, 521

Bhatia, A. K. \& DoscheK, G. A. 1992, Atomic Data Nuclear Data Tables, 52, 1

BhatiA, A. K. \& Doschek, G. A. 1993a, Atomic Data Nuclear Data Tables, 55, 315

Bhatia, A. K. \& DoscheK, G. A. 1993b, Atomic Data Nuclear Data Tables, 55, 281

Bhatia, A. K. \& DoscheK, G. A. 1993c, Atomic Data Nuclear Data Tables, 53, 195

Bhatia, A. K. \& DoscheK, G. A. 1995, Atomic Data Nuclear Data Tables, in press

Bhatia, A. K., Feldman, U., \& Seely, J. F. 1986, Atomic Data Nuclear Data Tables, 35, 319

Bhatia, A. K. \& Kastner, S. O. 1993a, ApJ, 408, 744

BhatiA, A. K. \& Kastner, S. O. 1993b, Atomic Data Nuclear Data Tables, 54, 133

Bhatia, A. K. \& Mason, H. E. 1980a, A\&A, 83, 380

Bhatia, A. K. \& MAson, H. E. 1980b, MNRAS, 190, 925

Bhatia, A. K. \& Mason, H. E. 1981, A\&A, 103, 324

Bhatia, A. K. \& Mason, H. E. 1986, A\&A, 155, 413

Bhatia, A. K., Seely, J. F., \& Feldman, U. 1987, Atomic Data Nuclear Data Tables, 36, 453

Brickhouse, N. S., Edgar, R., KaAstra, J., Kallman, T., Liedahl, D., Masai, K., Monsignori Fossi, B. C., Petre, R., Sanders, W., Savin, D. W., \& Stern, R. 1995a, Legacy, 6

Brickhouse, N. S., RaYmond, J. C., \& Smith, B. W. 1995b, ApJ, in press

Bromage, G. E., Cowan, R. D., \& Fawcett, B. C. 1977, Phys. Scripta, 15, 177 
Bromage, G. E., Cowan, R. D., \& Fawcett, B. C. 1978, Monthly Notices Roy. Astron. Soc., 183,19

Burgess, A. \& Tully, J. A. 1992, A\&A, 254, 436.

Christensen, R. B., Norcross, D. W., \& Pradhan, A. K. 1985, Physical Review A, 32, 93

Corliss, C. \& Sugar, J. 1985, in Spectroscopic Data for Iron, ed. W. L. Wiese

Cornille, M., Debau, J., loulergue, M., Bely-Debau, F., \& Faucher, P. 1992, A\&A, 259,669

Doschek, G. A., Feldman, U., van Hoosier, M. E., \& Bartoe, J. D. F. 1976, ApJS, 31, 417

Doyle, J. G. \& Keenan, T. P. 1992, A\&A, 264, 173

Dufton, P. L. \& Kingston, A. E. 1991, Physica Scripta, 43, 386

Dupree, A. K., Brickhouse, N. S., Doschek, G. A., Green, J. C., \& Raymond, J. C. 1993, ApJ, 418, L41

Edlen, B. 1983, Physica Scripta, 28, 483

EdLen, B. 1984, Physica Scripta, 30, 135

EdLEN, B. 1985, Physica Scripta, 31, 345

Fawcett, D. R. \& Mason, H. E. 1989, Atomic Data Nuclear Data Tables, 43, 245

Fawcett, B. C. \& Mason, H. E. 1991, Atomic Data Nuclear Data Tables, 47, 17

FLower, D. R. 1977a, A\&A, 54, 163

Flower, D. R. 1977b, A\&A, 56, 451

Froese, Fisher, C. \& Liu, B. 1986, Atomic Data Nuclear Data Tables, 34, 261

Gaetz, T. J. \& Salpeter, E. E. 1983, ApJS, 52, 155

Kastner, S. O., Neupert, W. M., \& Swartz, M. 1974, ApJ, 191, 261

Kato, T. 1976, ApJS, 30, 394

LANG, J, ED. 1994, Atomic Data Nuclear Data Tables, 57, no 1

LANDINI, M. \& Monsignoni Fossi,B. C. 1970, A\&A, 6,468

LANDinI, M. \& Monsignoni Fossi, B. C. 1990, A\&AS, 82, 229

LANDINI, M. \& MoNSIGNoRI FosSI, B. C. 1991, A\&AS, 91, 183

LANDINI, M \& MONSIGNORI FossI, B. C. 1993, A\&A, 275, L17

Loulergue, M., Mason, H. E., Nussbaumer, H., \& Storey, P. J. 1985, A\&A, 150, 246

MASAI, K. 1994, ApJ, 437, 770

MASON, H. E. 1975, MNRAS, 170, 651

Mason, H. E., DoscheK, G. A., Feldman, U., \& Bhatia, A. K. 1979, A\&A, 73, 74

Mason, H. E. \& Monsignori Fossi, B. C. 1994, Astron. Astroph. Rev., 6, 123

Mc, Kim, Melville, J. \& Berger, R. 1965, Planet. Space Sc., 13, 1131

Mewe, R. 1972, Solar Phys., 22, 459

Mewe, R., Groneschild, E. H. B. M., \& van den Oord, G. H. J. 1985, A\&AS, Ser., 62, 197

Mewe, R., KaAstra, J. S., Schrijver, C. J., van den Oord, \& Alkemade, F. J. M. 1995, $A \& A$, in press

Monsignori Fossi, B. C. \& LANDinI, M. 1991, Adv. Space Res, 11(1), 281

Monsignori Fossi, B. C. \& LANDini, M. 1994a, Solar Phys, 152, 81

Monsignori Fossi, B. C. \& Landini, M. 1994b, Atomic Data Nuclear Data Tables, 57, 125

MoNSIGNORI Fossi, B. C. \& LANDINI, M. 1994c, A\&A, 284, 900

Monsignori Fossi, B. C., Landini, M., Fruscione, A., \& Dupuis, J. 1995a, ApJ, in press

Monsignori Fossi, B. C., LANDini, M., Drake, J. J., \& CULly, S. L. 1995b, A\&A, in press

Muthethaler, H. P. \& Nussbaumer, H. 1976, A\&A, 48, 109 
Nussbaumer, H., \& Storey, P. J. 1979, A\&A, 74, 244

Pradhan. A. K. 1988, Atomic Data Nuclear Data Tables, 40, 335

RAYMOND, J. C. \& SMith, B. W. 1977, ApJS, 35, 419

Sampson, D. H., Zhang, H. L., \& Fontes, C. J. 1990, Atomic Data Nuclear Data Tables, 44, 210

Stern, R., WANG, R., BowYER, S. 1978, ApJS, 37, 195

Stern, R. A., Lemen, J. R., Schmitt, J. H. M. M., \& Pye, J. P. 1995, ApJ, in press

Tayal, S. S., \& Henry, R. J. W. 1986, ApJ, 302, 200

Tayal, S. S., Henry, R. J. W., \& Pradhan, A. 1987, ApJ, 319, 951

TAYal, S. S., \& HenRy, R. J. W. 1988, ApJ, 329, 1023

Thomas, R. J. \& NeUPert, W. M. 1994, ApJ, 91, 461

WIESE, W. L., SMith, M. W., \& Glennon, B. M. 1966, Atomic Trans. Prob., 1 NBS

Young, P. R., Mason, H. E., \& Thomas, R. J. 1995, in Proc. 3rd SOHO Workshop, in press

Zhang, H. \& SAMPson, D. H. 1992, Atomic Data Nuclear Data Tables, 52, 143 